\title{
Development of a Novel Intraoperative Neuromonitoring System Using an Accelerometer Sensor in Thyroid Surgery: A Porcine Model Study
}

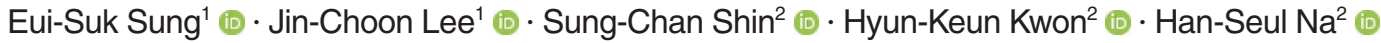 \\ Da-Hee Park ${ }^{1}$ (i) $\cdot$ Seong-Wook Choi' (i) $\cdot$ Jung-Hoon Ro ${ }^{3}$ (1) $\cdot$ Byung-Joo Lee ${ }^{2}$ (i) \\ ${ }^{I}$ Department of Otolaryngology-Head and Neck Surgery, Research Institute for Convergence of Biomedical Science and Technology, Pusan \\ National University Yangsan Hospital, Pusan National University College of Medicine, Yangsan; ${ }^{2}$ Department of Otolaryngology-Head and Neck \\ Surgery, Medical Research Institute, Pusan National University Hospital, Pusan National University College of Medicine, Busan; \\ ${ }^{3}$ Department of Biomedical Engineering, Pusan National University Hospital, Busan, Korea
}

Objectives. The sensitivity and positive predictive value of widely used intraoperative neuromonitoring (IONM) using electromyography (EMG) of the vocalis muscle in thyroid surgery are controversial. Thus, we developed a novel IONM system with an accelerometer sensor that uses the piezoelectric effect instead of EMG to detect laryngeal twitching. The objective of this study was to evaluate the feasibility and safety of this novel IONM system during thyroid surgery in a porcine model.

Methods. We developed an accelerometer sensor that uses the piezoelectric effect to measure laryngeal twitching in three dimensions. This novel accelerometer sensor was placed in the anterior neck skin (transcutaneous) or postcricoid area. Stimulus thresholds, amplitude, and latency of laryngeal twitching measured using the accelerometer sensor were compared to those measured through EMG of the vocalis muscle.

Results. The amplitudes of the accelerometer sensor at the anterior neck and postcricoid area were significantly lower than those of EMG because of differences in the measurement method used to evaluate laryngeal movement. However, no significant differences in stimulus thresholds between the EMG endotracheal tube and transcutaneous or postcricoid accelerometer sensors were observed.

Conclusion. Accelerometer sensors located at the anterior neck or postcricoid area were able to identify laryngeal twitching. The stimulus intensity measured with these sensors was equivalent to that from conventional vocalis EMG. Our novel IONM system with an accelerometer sensor that checks changes in surface acceleration can be an alternative to EMG of the vocalis muscle for IONM in the future.

Keywords: Neuromonitoring; Accelerometer Sensor; Electromyography; Recurrent Laryngeal Nerve; Thyroidectomy

\footnotetext{
- Received March 18, 2019

Revised May 9, 2019

Accepted May 20, 2019

- Corresponding author: Byung-Joo Lee

Department of Otolaryngology-Head and Neck Surgery, Medical Research Institute, Pusan National University Hospital, Pusan National University College of Medicine, 179 Gudeok-ro, Seo-gu, Busan 49241, Korea Tel: +82-51-240-7675, Fax: +82-51-246-8668

E-mail: voiceleebj@gmail.com
}

\section{INTRODUCTION}

Recurrent laryngeal nerve (RLN) injury during thyroid surgery can affect a patient's quality of life and result in legal predicaments $[1,2]$. Unilateral vocal fold palsy (VFP) may induce hoarseness, voice fatigue, and aspiration, whereas bilateral VFP may require tracheostomy [3]. The rates of temporary and persistent VFP after thyroidectomy are reported to be $9.8 \%$ and $2.3 \%$, respectively (range, $2.3 \%-26 \%$ ) [4]. Therefore, RLN preservation is one of the most important tasks during thyroid sur-

Copyright @ 2019 by Korean Society of Otorhinolaryngology-Head and Neck Surgery.

This is an open-access article distributed under the terms of the Creative Commons Attribution Non-Commercial License (http://creativecommons.org/licenses/by-nc/4.0)

which permits unrestricted non-commercial use, distribution, and reproduction in any medium, provided the original work is properly cited. 
gery. Although lower rates of RLN palsy are reported with visual nerve identification than without visualization [5-7], confirmation of functional integrity of the RLN by visual identification alone is close to impossible as most injuries cause functional abnormalities without structural changes to the nerve appearance. Because most RLN injuries are functional, such as traction, compression, thermal injury, and ischemia, rather than structural. Therefore, intraoperative neuromonitoring (IONM) is widely used for verification of both functional and structural integrity of the RLN during thyroid surgery [8].

The IONM system uses an electrode attached to an endotracheal tube to perform an electromyography (EMG) of the vocalis muscle, which can reveal nerve mapping, nerve identification, nerve functional integrity, a prediction of postoperative VFP, and the mechanism of RLN palsy [8-11]. However, the current IONM system has several problems. A nerve stimulator is repeatedly used for nerve identification during surgery but is cumbersome, time-consuming [12]. Thus, an attachable and detachable nerve stimulator with metallic, endoscopic, or robotic surgical instruments and an energy device surgical instrument are required [13-15]. Another problem is the wide range of sensitivity and positive predictive values (PPV) of recording part to measure EMG of vocalis muscle. Several methods have been reported to overcome this problem, including the Tri-Vantage EMG endotracheal tube, subperichondrial needle electrodes, postcricoid electrodes, transcartilage electrodes, and transcutaneous electrodes [16-22]. However, all of these methods have problems related with the EMG method. For example, at least two electrodes should be attached to the corresponding muscle [12]. Because EMG measures the electrical phase difference, it can be affected by saliva or sputum in the larynx, and EMG may not work during use of an electric surgical unit [12].

Contact between the vocal fold and the electrode is important, and variations in sensitivity and PPV are often caused by malposition of the EMG endotracheal tube. If a loss of signal (LOS) occurs during surgery, the surgeon confirms the laryngeal twitch using fingers [8]. Even with LOS, the possibility of RLN injury is low if laryngeal twitching is present. A pressure sensor using the piezoelectric effect can also be used to evaluate mus-

\section{H I G G H L I G G H T S}

- The risk of nerve damage may occur due to false-negative or positive responses on conventional intraoperative neuromonitoring (IONM).

- We developed an IONM system using an accelerometer sensor to detect muscle twitching in three dimensions.

- It is to measure the change of acceleration using a piezo-electric effect instead of electromyography.

- The application of IONM using an accelerometer sensor during surgery is noninvasive, safe, and feasible. cle twitching, and a novel IONM system that uses a pressure sensor to measure muscle twitching instead of EMG has been proposed [13].

The piezoelectric sensor converts changes in pressure, temperature, acceleration, and force into an electrical charge and has been used in medical devices because of its safety. A conventional piezoelectric sensor measures two-dimensional surface pressure changes. An accelerometer sensor using the piezoelectric effect can measure and record a variety of dynamic mechanical changes including shock, vibration, and the motion of an object in three dimensions.

The evaluation of laryngeal twitching would be more effective with three-dimensional measurements than with two-dimensional measurements. Therefore, we developed a novel accelerometer sensor that could measure the three-dimensional motion of laryngeal twitching in contact with the anterior neck skin and postcricoid area. After stimulating the RLN, the amplitude and latency of laryngeal twitching was measured by the accelerometer sensor and compared with those using EMG of the vocalis muscle to confirm the applicability of our novel IONM system. The objective of the present study was to evaluate the feasibility and safety of our novel IONM system during thyroid surgery in a porcine model.

\section{MATERIALS AND METHODS}

\section{Animals}

The experimental protocol was approved by Institutional Animal Care and Use Committee of Pusan National University Yangsan Hosptial. The experiment was carried out using eight RLNs in four Yorkshire-Landrace-Duroc female pigs $(30-40 \mathrm{~kg})$ that underwent thyroidectomy. The equipment and anesthesia protocol were as follows: the pigs were sedated with an intramuscular injection of ketamine hydrochloride $(20 \mathrm{mg} / \mathrm{kg})$ and xylazine $(2 \mathrm{mg} / \mathrm{kg})$; anesthesia was maintained with inhaled 3\% isoflurane; all pigs were intubated with a standard reinforced EMG endotracheal tube $(7.0 \mathrm{~mm}$; Medtronic Xomed, Jacksonville, FL, USA) under a nerve integrity monitor (NIM; Response 3.0 System, Medtronic Xomed); simultaneously, an accelerometer sensor was attached to the anterior neck skin at the upper margin of both laminas of the thyroid cartilage using Tegaderm (Fig. 1) or inserted into cricoid cartilage in the hypopharynx above the esophageal inlet (Fig. 2); the postcricoid sensor was placed with a flexible fiberscope through visualization; thereafter, the location of the EMG endotracheal tube was confirmed with a flexible fiberscope and thyroid surgery was performed.

The largest amplitudes of EMG and the accelerometer sensor were recorded, and the latencies of EMG and the accelerometer sensor were registered at the largest amplitudes. All pigs were monitored using a NIM and a hand-made accelerometer sensor monitor.The pigs were euthanized at the end of the experiment. 

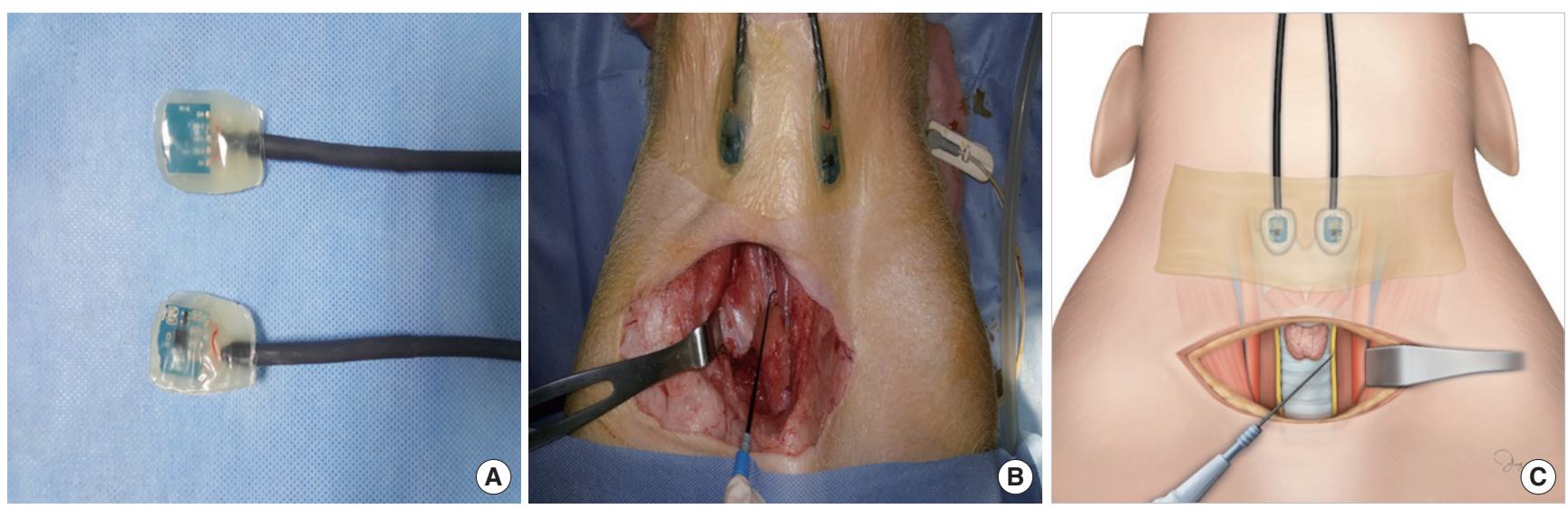

Fig. 1. (A) Photograph of a transcutaneous accelerometer sensor. (B) Surgical field of dissection on the pig, demonstrating the accelerometer sensor attached to the anterior neck skin. (C) Illustration of surgical field with the transcutaneous accelerometer sensor.
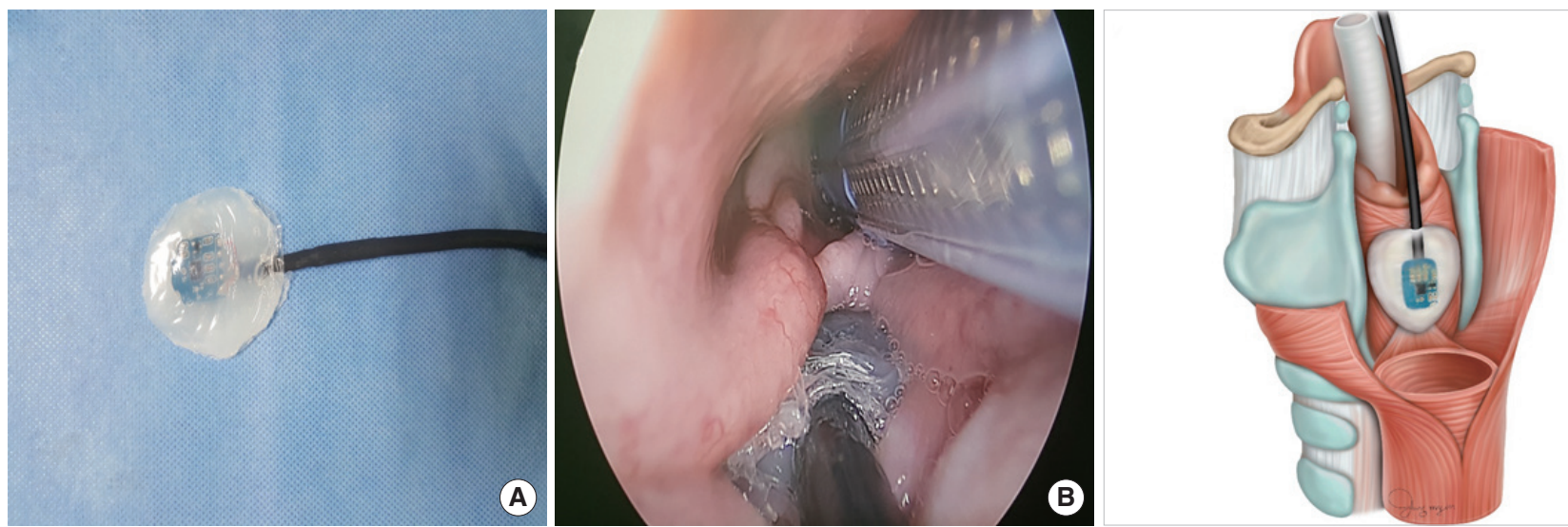

Fig. 2. (A) Photograph of a postcricoid accelerometer sensor. (B) Intraoperative endoscopic view of larynx on the pig, demonstrating the accelerometer sensor attached postcricoid area. (C) Illustration of the postcricoid accelerometer sensor placement.

\section{Accelerometer sensor}

The novel accelerometer sensor was modified to measure the change in acceleration using the piezoelectric effect to detect muscle twitching (Figs. 1A, 2A). A transcutaneous sensor was attached to the skin of the upper margin of the laminas of the thyroid cartilage and sensed the changes in acceleration due to movement of the vocalis muscle (Fig. 1B, C). A postcricoid sensor was located behind the cricoid cartilage in the hypopharynx, and contacts were placed around the posterior cricoarytenoid (PCA) muscles (Fig. 2B, C). The sensor was a surface-type device and attached to the skin or around muscle without the insertion of a needle electrode into the muscle. Acceleration was generated by muscle twitching due to nerve stimulus, and the accelerometer sensor measured the amplitude and latency of the muscle movement.

The stimulation threshold was defined as the stimulus value at which the first response appears in the RLN. The average amplitude and latency time of EMG of the vocalis muscle and the accelerometer sensor at the anterior neck skin (transcutaneous) or postcricoid area were compared. We assessed the amplitude, latency, and stimulus thresholds of EMG and the accelerometer sensor in the eight RLNs of four pigs (Tables 1,2).

\section{Statistical analysis}

Statistical analyses were performed using the Mann-Whitney $U$ test with IBM SPSS ver. 21.0 (IBM Corp., Armonk, NY, USA). A $P$-value $<0.05$ was considered statistically significant.

\section{RESULTS}

After stimulating the RLN, stimulus thresholds, amplitudes, and latencies of EMG of the vocalis muscle and the transcutaneous accelerometer sensor were evaluated (Table 1). The accelerometer sensor was deemed feasible and safe for use in this experiment. The mean amplitudes of EMG and the transcutaneous accelerometer sensor were $499.33 \pm 288.60 \mu \mathrm{V}$ and $10.42 \pm 4.25 \mu \mathrm{V}$, respectively, and the mean latencies were $5.30 \pm 0.51 \mathrm{~ms}$ and 
Table 1. Analysis of the amplitude and latency of the EMG of vocalis muscle and transcutaneous accelerometer sensor depending on the value of the RLN stimulus in pigs

\begin{tabular}{|c|c|c|c|c|c|c|c|c|}
\hline \multirow{2}{*}{ Stimulus $(\mathrm{mA})$} & \multicolumn{3}{|c|}{ EMG } & \multicolumn{3}{|c|}{ Transcutaneous accelerometer sensor } & \multicolumn{2}{|c|}{$P$-value ${ }^{\text {a) }}$} \\
\hline & Response (n) & Amplitude $(\mu \mathrm{V})$ & Latency (ms) & Response (n) & Amplitude $(\mu \mathrm{V})$ & Latency (ms) & Amplitude & Latency \\
\hline 0.3 & 8 & $358.37 \pm 234.60$ & $5.28 \pm 0.54$ & 8 & $7.72 \pm 3.04$ & $35.50 \pm 11.90$ & & \\
\hline 0.5 & 8 & $467.62 \pm 294.92$ & $5.31 \pm 0.54$ & 8 & $9.92 \pm 4.51$ & $34.45 \pm 12.61$ & & \\
\hline 1.0 & 8 & $514.25 \pm 240.39$ & $5.31 \pm 0.54$ & 8 & $10.97 \pm 4.16$ & $34.55 \pm 12.70$ & & \\
\hline 1.5 & 8 & $531.87 \pm 351.03$ & $5.31 \pm 0.54$ & 8 & $10.95 \pm 4.18$ & $34.70 \pm 12.57$ & & \\
\hline 2.0 & 8 & $551.25 \pm 315.34$ & $5.31 \pm 0.54$ & 8 & $11.17 \pm 4.40$ & $34.05 \pm 12.42$ & & \\
\hline 3.0 & 8 & $572.62 \pm 319.75$ & $5.31 \pm 0.54$ & 8 & $11.77 \pm 5.06$ & $33.75 \pm 12.73$ & & \\
\hline Average & 48 & $499.33 \pm 288.60$ & $5.30 \pm 0.51$ & 48 & $10.42 \pm 4.25$ & $34.50 \pm 11.82$ & $<0.001$ & $<0.001$ \\
\hline
\end{tabular}

Values are presented as mean \pm standard deviation.

EMG, electromyography; RLN, recurrent laryngeal nerve.

a) $P$-value indicates the result of the comparison test for the mean response values of the amplitude and latency between the EMG and surface accelerometer sensor depending on stimulus value.

Table 2. Analysis of the amplitude and latency of the EMG of vocalis muscle and postcricoid accelerometer sensor depending on the value of the RLN stimulus in pigs

\begin{tabular}{|c|c|c|c|c|c|c|c|c|}
\hline \multirow{2}{*}{ Stimulus (mA) } & \multicolumn{3}{|c|}{ EMG } & \multicolumn{3}{|c|}{ Postcricoid accelerometer sensor } & \multicolumn{2}{|c|}{$P$-value ${ }^{a)}$} \\
\hline & Response (n) & Amplitude $(\mu \mathrm{V})$ & Latency (ms) & Response (n) & Amplitude $(\mu \mathrm{V})$ & Latency (ms) & Amplitude & Latency \\
\hline 0.3 & 4 & $134.75 \pm 29.72$ & $5.06 \pm 0.51$ & 4 & $16.94 \pm 5.35$ & $6.92 \pm 0.94$ & & \\
\hline 0.5 & 8 & $182.85 \pm 98.36$ & $4.92 \pm 0.42$ & 8 & $17.47 \pm 5.35$ & $6.72 \pm 0.76$ & & \\
\hline 1.0 & 8 & $301.85 \pm 169.11$ & $4.92 \pm 0.42$ & 8 & $23.74 \pm 9.68$ & $6.70 \pm 0.75$ & & \\
\hline 1.5 & 8 & $325.14 \pm 131.71$ & $4.92 \pm 0.42$ & 8 & $22.39 \pm 8.06$ & $6.61 \pm 0.66$ & & \\
\hline 2.0 & 8 & $364.71 \pm 139.84$ & $4.92 \pm 0.42$ & 8 & $22.39 \pm 8.06$ & $6.67 \pm 0.53$ & & \\
\hline 3.0 & 8 & $370.42 \pm 143.08$ & $4.92 \pm 0.42$ & 8 & $24.51 \pm 8.63$ & $6.60 \pm 0.62$ & & \\
\hline Average & 44 & $291.12 \pm 149.32$ & $4.94 \pm 0.40$ & 44 & $21.51 \pm 7.88$ & $6.69 \pm 0.66$ & $<0.001$ & 0.790 \\
\hline
\end{tabular}

Values are presented as mean \pm standard deviation.

EMG, electromyography; RLN, recurrent laryngeal nerve.

a) $P$-value indicates the result of the comparison test for the mean response values of the amplitude and latency between the EMG and surface accelerometer sensor depending on stimulus value.

$34.50 \pm 11.82 \mathrm{~ms}$, respectively. EMG and the transcutaneous accelerometer sensor showed a response to $0.3 \mathrm{~mA}$ stimulation in all RLNs. Table 2 presents the results of EMG of the vocalis muscle and the postcricoid accelerometer sensor. The mean amplitudes of EMG and the postcricoid accelerometer sensor were $291.12 \pm 149.32 \mu \mathrm{V}$ and $21.51 \pm 7.88 \mu \mathrm{V}$, respectively, and the mean latencies were $4.94 \pm 0.40 \mathrm{~ms}$ and $6.69 \pm 0.66 \mathrm{~ms}$, respectively. EMG and the postcricoid accelerometer sensor showed a response to a $0.3 \mathrm{~mA}$ stimulus in four of the eight RLNs and a response to a $0.5 \mathrm{~mA}$ stimulus in all RLNs.

No differences in the stimulus intensity threshold between EMG and the transcutaneous/postcricoid accelerometer sensors were observed. The mean amplitude difference between EMG and the transcutaneous accelerometer sensor was $488 \mu \mathrm{V}$, and the mean latency time difference between them was $32 \mathrm{~ms}$. Significant differences in the amplitude and latency between EMG and the transcutaneous accelerometer sensor $(P<0.001, P<$ 0.001 , respectively) were observed and are shown in Table 1 . However, a simple comparison of the amplitude difference would not be appropriate because of the measurement differ- ences. A lag in the response by 0.032 seconds was clinically marginal during thyroidectomy. In addition, no difference in the latencies between EMG and the postcricoid accelerometer sensor was observed.

\section{DISCUSSION}

IONM during thyroid surgery has been used worldwide [23], and a recent meta-analysis reported that IONM could decrease both temporary and permanent VFP rates [24]. However, other studies have reported that IONM during thyroid surgery is controversial because VFP was not significantly reduced [23,25-30].

Conventional IONM with EMG has problems on both the stimulating side, which are mentioned in the introduction, and the recording side [12]. Malposition of electrodes on the EMG endotracheal tube is the most common cause of LOS, which can result in dysfunction of IONM $[8,31,32]$. EMG can only be measured if both electrodes are touching the vocal fold during surgery $[33,34]$, and adjustments to the EMG endotracheal tube 
intraoperatively due to poor contact with the vocal cords are reported to occur in $10 \%$ of patients undergoing thyroid surgery [32]. The criteria for normal vocal cord EMG amplitude are not clear, and the EMG endotracheal tube cannot be used if a tracheostomy is needed or general anesthesia is not available. IONM is unavailable during surgery unless an EMG endotracheal tube is used before surgery. If saliva or sputum is present around the tube's electrode or electro-cauterization is used, an error in the EMG measurement occurs.

Although IONM using EMG endotracheal tube has a highly negative predictive value of $92 \%-100 \%$, the sensitivity and PPV have a variable range of $10 \%-90 \%$ [23]; this implies that there is a high possibility that contact between electrodes on the EMG endotracheal tube and vocal cords has changed owing to neck extension or surgical manipulation [11,31,34]. Contact between the target muscle and electrode is very important to detect muscle movement and obtain accurate IONM results. Conventional IONM using EMG endotracheal tube is troublesome and inefficient because of the necessity to confirm proper positioning, as mentioned above [35]. Several studies have reported the use of thyroid cartilage electrode EMG, transcutaneous surface electrode EMG, transcartilage surface electrode EMG by Wu et al. [21,22], and PCA muscle EMG by Liddy et al. [20] to overcome the disadvantages of the conventional EMG endotracheal tube [35]. However, these approaches are EMG-based IONM and cannot completely overcome the above-mentioned shortcomings of EMG.

The method of detecting PCA muscle twitching using fingers as a troubleshooting maneuver for LOS is well-known $[8,36]$. We approached this study with the hypothesis that there may be a way to detect muscle movements more sensitively and quantitatively using a pressure sensor instead of fingers. We proposed a novel IONM system using a piezoelectric surface-pressure sensor that could measure muscle twitching instead of using EMG [13]. The previous piezoelectric surface-pressure sensor sensed two-dimensional muscle movement. However, evaluation of laryngeal twitching using three-dimensional measurements is more effective. The accelerometer sensor measures muscle twitching in three dimensions using the change of acceleration. The accelerometer sensor is sensitive to gravity or the opposite direction, because it is related to gravitational acceleration; consequently, it can detect laryngeal muscle twitching more sensitively.

Our results show no difference in the stimulus intensity threshold between the accelerometer sensors and EMG, and the different amplitudes between them were due to differences in the measurement method. The transcutaneous accelerometer sensor response lag of 0.032 seconds compared to EMG was not clinically meaningful. The latencies of the postcricoid accelerometer sensor were equivalent to those of EMG. If the dissection of the upper skin flap in humans is raised too high or the superior pole is dissected, the transcutaneous accelerometer sensor may not be in contact with the thyroid cartilage and accurate nerve monitoring may not be possible. And the transcutaneous sensor is also not suitable for surgery that requires very large incisions [22]. However, the transcutaneous sensor can be reattached if it is dislodged from the skin. The transcutaneous sensor is considered more stable than the EMG endotracheal tube, because the anatomical relationship between the transcutaneous sensor and the vocalis muscle is not affected by the surgical manipulation [35]. The sensor should be firmly attached to the skin by Tegaderm to prevent contact failure [22]. Postcricoid EMG electrodes provide similar sensitivity to electrodes on the EMG endotracheal tube [8,37], and postcricoid EMG seems to be the best reflection of PCA muscle movement and a better reflection of the abduction movement of the vocal cords [37]. Therefore, we consider the postcricoid accelerometer sensor has an advantage similar to postcricoid EMG.

EMG records muscle movement by measuring the difference of action potential between two electrodes. Thus, if an electrode is not in contact with the vocal cords due to the positional change of a patient or the surgical procedure, EMG will not accurately assess muscle motion. In addition, EMG may be affected by the presence of sputum or saliva around the electrode. Whereas an accelerometer sensor can evaluate muscle twitching by being in only partial contact with the skin or mucosal surface, and its measurement of surface pressure changes is unlikely to be affected by the presence of sputum or saliva. Therefore, the accelerometer sensor should overcome the disadvantages of IONM using EMG.

This study has several limitations. First, the amplitude of the accelerometer sensor was very low and significantly different from that of EMG. However, the difference in amplitude was due to differences in the measurement method. However, the method of amplifying the amplitudes is needed in future. Second, conventional IONM systems can predict nerve injury by using the change of EMG amplitude or latency time caused by traction or compression, which is a common cause of nerve damage. In order for the accelerometer sensor to replace the IONM system using EMG measurements, it must be able to predict nerve damage by the changes of amplitude or latency according to the nerve injury mechanism, and further research is needed. Third, this study was a small sample animal experiment using a porcine model. A large-scale human study is required to certify the feasibility and safety of this novel device.

In conclusion, the accelerometer sensors located at the anterior neck or postcricoid area were able to identify laryngeal twitching. The stimulus threshold intensity with the accelerometer sensor, which measured laryngeal twitching, was equivalent to that of the conventional vocalis EMG. Our novel IONM system that uses an accelerometer sensor to check the change of surface acceleration can be an alternative to IONM systems using EMG of the vocalis muscle. 


\section{CONFLICT OF INTEREST}

No potential conflict of interest relevant to this article was reported.

\section{ORCID}

$\begin{array}{ll}\text { Eui-Suk Sung } & \text { https://orcid.org/0000-0001-8752-3426 } \\ \text { Jin-Choon Lee } & \text { https://orcid.org/0000-0002-5629-4277 } \\ \text { Sung-Chan Shin } & \text { https://orcid.org/0000-0003-2329-0648 } \\ \text { Hyun-Keun Kwon } & \text { https://orcid.org/0000-0003-4089-8639 } \\ \text { Han-Seul Na } & \text { https://orcid.org/0000-0002-0118-3887 } \\ \text { Da-Hee Park } & \text { https://orcid.org/0000-0001-7466-2793 } \\ \text { Seong-Wook Choi } & \text { https://orcid.org/0000-0002-2459-462X } \\ \text { Jung-Hoon Ro } & \text { https://orcid.org/0000-0002-5203-5956 } \\ \text { Byung-Joo Lee } & \text { https://orcid.org/0000-0001-7091-6688 }\end{array}$

\section{AUTHOR CONTRIBUTIONS}

Conceptualization: BJL. Data curation: HSN, DHP, SWC. Formal analysis: JCL, HGK. Methodology: JHR. Project administration: SCS. Visualization: JHR. Writing - original draft: ESS. Writing - review \& editing: ESS, BJL.

\section{REFERENCES}

1. Haugen BR, Alexander EK, Bible KC, Doherty GM, Mandel SJ, Nikiforov YE, et al. 2015 American Thyroid Association Management Guidelines for Adult Patients with Thyroid Nodules and Differentiated Thyroid Cancer: The American Thyroid Association Guidelines Task Force on Thyroid Nodules and Differentiated Thyroid Cancer. Thyroid. 2016 Jan;26(1):1-133.

2. Chandrasekhar SS, Randolph GW, Seidman MD, Rosenfeld RM, Angelos P, Barkmeier-Kraemer J, et al. Clinical practice guideline: improving voice outcomes after thyroid surgery. Otolaryngol Head Neck Surg. 2013 Jun;148(6 Suppl):S1-37.

3. Munch S, deKryger L. A piece of my mind. Moral wounds: complicated complications. JAMA. 2001 Mar;285(9):1131-2.

4. Jeannon JP, Orabi AA, Bruch GA,Abdalsalam HA, Simo R. Diagnosis of recurrent laryngeal nerve palsy after thyroidectomy: a systematic review. Int J Clin Pract. 2009 Apr;63(4):624-9.

5. Riddell V. Thyroidectomy: prevention of bilateral recurrent nerve palsy. Results of identification of the nerve over 23 consecutive years (1946-69) with a description of an additional safety measure. Br J Surg. 1970 Jan;57(1):1-11.

6. Hermann M, Alk G, Roka R, Glaser K, Freissmuth M. Laryngeal recurrent nerve injury in surgery for benign thyroid diseases: effect of nerve dissection and impact of individual surgeon in more than 27,000 nerves at risk. Ann Surg. 2002 Feb;235(2):261-8.

7. Chiang FY,Wang LF, Huang YF, Lee KW, Kuo WR. Recurrent laryngeal nerve palsy after thyroidectomy with routine identification of the recurrent laryngeal nerve. Surgery. 2005 Mar;137(3):342-7.

8. Randolph GW, Dralle H; International Intraoperative Monitoring Study Group, Abdullah H, Barczynski M, Bellantone R, et al. Elec- trophysiologic recurrent laryngeal nerve monitoring during thyroid and parathyroid surgery: international standards guideline statement. Laryngoscope. 2011 Jan;121 Suppl 1:S1-16.

9. Terris DJ, Snyder S, Carneiro-Pla D, InabnetWB 3rd, Kandil E, Orloff L, et al. American Thyroid Association statement on outpatient thyroidectomy. Thyroid. 2013 Oct;23(10):1193-202.

10. Chen AY, Bernet VJ, Carty SE, Davies TF, Ganly I, Inabnet WB 3rd, et al. American Thyroid Association statement on optimal surgical management of goiter.Thyroid. 2014 Feb;24(2):181-9.

11. Chiang FY, Lee KW, Chen HC, Chen HY, Lu IC, Kuo WR, et al. Standardization of intraoperative neuromonitoring of recurrent laryngeal nerve in thyroid operation. World J Surg. 2010 Feb;34(2): 223-9.

12. Sung ES, Lee BJ. Development of the novel intraoperative neuromonitoring for thyroid surgery. Int JThyroid. 2018;11(2):109-16.

13. Sung ES, Lee JC, Shin SC, Kwon HG, Kim MS, Kim DJ, et al. Development of a novel intraoperative neuromonitoring system using a surface pressure sensor to detect muscle movement: a rabbit model study. Clin Exp Otorhinolaryngol. 2019 May;12(2):217-23.

14. Sung ES, Lee JC, Kim SH, Shin SC, Jung DW, Lee BJ. Development of an attachable endoscopic nerve stimulator for intraoperative neuromonitoring during endoscopic or robotic thyroidectomy. Otolaryngol Head Neck Surg. 2018 Mar;158(3):465-8.

15. Shin SC, Sung ES, Choi SW, Kim SD, Jung DW, Kim SH, et al. Feasibility and safety of nerve stimulator attachment to energy-based devices: a porcine model study. Int J Surg. 2017 Dec;48:155-9.

16. Khan A, Pearlman RC, Bianchi DA, Hauck KW. Experience with two types of electromyography monitoring electrodes during thyroid surgery. Am J Otolaryngol. 1997 Mar-Apr;18(2):99-102.

17. Tschopp KP, Gottardo C. Comparison of various methods of electromyographic monitoring of the recurrent laryngeal nerve in thyroid surgery. Ann Otol Rhinol Laryngol. 2002 Sep;111(9):811-6.

18. Rea JL, Khan A. Clinical evoked electromyography for recurrent laryngeal nerve preservation: use of an endotracheal tube electrode and a postcricoid surface electrode. Laryngoscope. 1998 Sep;108(9): 1418-20.

19. Marcus B, Edwards B, Yoo S, Byrne A, Gupta A, Kandrevas J, et al. Recurrent laryngeal nerve monitoring in thyroid and parathyroid surgery: the University of Michigan experience. Laryngoscope. 2003 Feb;113(2):356-61.

20. Liddy W, Barber SR, Lin BM, Kamani D, Kyriazidis N, Lawson B, et al. Monitoring of the posterior cricoarytenoid muscle represents another option for neural monitoring during thyroid surgery: normative vagal and recurrent laryngeal nerve posterior cricoarytenoid muscle electromyographic data. Laryngoscope. 2018 Jan;128(1): 283-9.

21. Wu CW, Chiang FY, Randolph GW, Dionigi G, Kim HY, Lin YC, et al. Feasibility of intraoperative neuromonitoring during thyroid surgery using transcartilage surface recording electrodes. Thyroid. 2018 Nov;28(11):1508-16.

22. Wu CW, Chiang FY, Randolph GW, Dionigi G, Kim HY, Lin YC, et al.Transcutaneous recording during intraoperative neuromonitoring in thyroid surgery. Thyroid. 2018 Nov;28(11):1500-7.

23. Dralle H, Sekulla C, Lorenz K, Brauckhoff M, Machens A; German IONM Study Group. Intraoperative monitoring of the recurrent laryngeal nerve in thyroid surgery. World J Surg. 2008 Jul;32(7):135866.

24. Bai B, Chen W. Protective effects of intraoperative nerve monitoring (IONM) for recurrent laryngeal nerve injury in thyroidectomy: meta-analysis. Sci Rep. 2018 May;8(1):7761.

25. Sanabria A, Ramirez A, Kowalski LP, Silver CE, Shaha AR, Owen RP, et al. Neuromonitoring in thyroidectomy: a meta-analysis of effectiveness from randomized controlled trials. Eur Arch Otorhinolaryngol. 2013 Aug;270(8):2175-89. 
26. Lifante JC, McGill J, Murry T, Aviv JE, Inabnet WB 3rd. A prospective, randomized trial of nerve monitoring of the external branch of the superior laryngeal nerve during thyroidectomy under local/regional anesthesia and IV sedation. Surgery. 2009 Dec;146(6):116773.

27. Barczynski M, Konturek A, Cichon S. Randomized clinical trial of visualization versus neuromonitoring of recurrent laryngeal nerves during thyroidectomy. Br J Surg. 2009 Mar;96(3):240-6.

28. Higgins TS, Gupta R, Ketcham AS, Sataloff RT, Wadsworth JT, Sinacori JT. Recurrent laryngeal nerve monitoring versus identification alone on post-thyroidectomy true vocal fold palsy: a meta-analysis. Laryngoscope. 2011 May;121(5):1009-17.

29. Dionigi G, Boni L, Rovera F, Bacuzzi A, Dionigi R. Neuromonitoring and video-assisted thyroidectomy: a prospective, randomized casecontrol evaluation. Surg Endosc. 2009 May;23(5):996-1003.

30. Chan WF, Lo CY. Pitfalls of intraoperative neuromonitoring for predicting postoperative recurrent laryngeal nerve function during thyroidectomy.World J Surg. 2006 May;30(5):806-12.

31. Chiang FY, Lu IC, KuoWR, Lee KW, Chang NC,Wu CW.The mechanism of recurrent laryngeal nerve injury during thyroid surgery: the application of intraoperative neuromonitoring. Surgery. 2008 Jun;143(6):743-9.

32. Dionigi G, Bacuzzi A, Boni L, Rovera F, Dionigi R. What is the learn- ing curve for intraoperative neuromonitoring in thyroid surgery? Int J Surg. 2008;6 Suppl 1:S7-12.

33. Kim HY,Tufano RP, Randolph G, Barczynski M,Wu CW, Chiang FY, et al. Impact of positional changes in neural monitoring endotracheal tube on amplitude and latency of electromyographic response in monitored thyroid surgery: results from the porcine experiment. Head Neck. 2016 Apr;38 Suppl 1:E1004-8.

34. Wu CW,Wang MH, Chen CC, Chen HC, Chen HY,Yu JY, et al. Loss of signal in recurrent nerve neuromonitoring: causes and management. Gland Surg. 2015 Feb;4(1):19-26.

35. Chiang FY, Lu IC, Chang PY, Dionigi G, Randolph GW, Sun H, et al. Comparison of EMG signals recorded by surface electrodes on endotracheal tube and thyroid cartilage during monitored thyroidectomy. Kaohsiung J Med Sci. 2017 Oct;33(10):503-9.

36. Randolph GW, Kobler JB, Wilkins J. Recurrent laryngeal nerve identification and assessment during thyroid surgery: laryngeal palpation.World J Surg. 2004 Aug;28(8):755-60.

37. Puram SV, Chow H, Wu CW, Heaton JT, Kamani D, Gorti G, et al. Posterior cricoarytenoid muscle electrophysiologic changes are predictive of vocal cord paralysis with recurrent laryngeal nerve compressive injury in a canine model. Laryngoscope. 2016 Dec;126(12): 2744-51. 